\title{
APPLICATION OF SBAS PSEUDORANGE AND CARRIER PHASE SIGNALS TO PRECISE INSTANTANEOUS SINGLE-FREQUENCY POSITIONING
}

\author{
Jacek PAZIEWSKI *, Paweł WIELGOSZ and Marta KRUKOWSKA
}

\author{
University of Warmia and Mazury in Olsztyn, Oczapowskiego 2, Poland \\ *Corresponding author's e-mail: jacek.paziewski@uwm.edu.pl
}

(Received February 2013, accepted May 2013)

\begin{abstract}
This research evaluates the potential benefits of the tightly combined processing of a global navigation satellite system together with the additional ranging observations from a satellite based augmentation system. In specific, the experiment presents performance of precise instantaneous single-frequency positioning based on European Galileo and EGNOS navigation systems. Due to currently low number of Galileo satellites, the test observational data were obtained with hardware GNSS signal simulator. All calculations were performed with in-house developed software - GINPOS.

The results show that it is possible to obtain improvement in the accuracy and reliability of single-frequency precise positioning when including observations from SBAS systems. However, one must take into account that at middle latitudes EGNOS satellites are observed at low elevations what results in higher atmospheric errors affecting its signals.
\end{abstract}

KEYWORDS: GNSS, Galileo, SBAS, EGNOS, precise satellite positioning

\section{INTRODUCTION}

The potential of the application of GNSS technology in geodynamic studies is thoroughly recognized. Satellite positioning offers variety of methods, which differ in terms of accuracy, reliability and required length of an observational session. Precise, relative positioning is considered to be the method, which guarantees the highest accuracy and reliability of the solution (Kapłon and Cacoń, 2009; Cacoń et al., 2010; Schenk et al., 2010). On the other hand, development of new global satellite navigation and augmentation systems (e.g. Galileo, BeiDou, QZSS) as well as modernization of the existing ones (e.g. GPS, GLONASS, EGNOS) gives opportunities for advancement in the positioning algorithms (Verhagen, 2002; Zhao et al., 2005; Paziewski 2012).

The main mission of the SBAS (Satellite Based Augmentation System) systems such as EGNOS is to provide integrity information as well as the differential correction data in order to enhance the performance of positioning navigation services (ESA, 2006). What is more, these systems transmit also carrier phase and pseudorange signals (so called a range service), which can be applied in precise positioning together with observations from global satellite positioning systems such as GPS, Galileo or GLONASS. Presently three of the EGNOS satellites transmit carrier phase and pseudorange observations on L1 frequency, which is overlapping with Galileo E1 and GPS L1 frequency. In the future, dualfrequency (L1\&L5) observations will be provided.
Despite this, the range service is seldom used to enhance precise positioning in surveying practice. Sauer and Ochieng (2002) presented the first results of application of GPS and EGNOS in precise kinematic positioning. Their research indicated that the quality of EGNOS pseudorange observations was lower than GPS. What is more, studies show low quality of SBAS satellites ephemeridal data - orbits and clocks (Wanninger and Wallstab-Freitag, 2007).

Also, Galileo - the first European global satellite navigation system is still under development. Presently, there are four IOV (In Orbit Validation) satellites (January 2013). Two earlier satellites, GIOVE-A and GIOVE-B, which served during the test and implementation phase were decommissioned. However, this number of operational Galileo satellites is still insufficient to conduct research on combined processing of signals collected from Galileo+EGNOS satellites based on real observational data.

Nevertheless, the aim of the presented research is to study the potential benefits of the application of the SBAS carrier phase and pseudorange observations in the precise positioning. The research assume using single-frequency signals from full constellations of Galileo and EGNOS satellites in instantaneous (single-epoch) mode. We limited our studies to L1 signal, because only single-frequency observations are currently provided by EGNOS system. Overlapping frequencies allow for tight coupling of the observations from these two systems. It may be assumed that additional signals from SBAS satellites 
will have positive impact especially on the carrier phase ambiguity resolution, which is crucial in the precise, relative positioning. It is likely that availability, reliability and accuracy of the precise positioning will benefit from the introduction of additional geostationary satellite signals (Sauer and Ochieng, 2002).

Performance of the combined, single-frequency precise Galileo+EGNOS positioning was evaluated on the basis of simulated data. This allowed for overcoming of the problem of the low quality of the operational EGNOS orbits and clocks, as well as unsatisfactory number of Galileo satellites.

\section{METHODOLOGY}

All calculations were performed applying GINPOS (GNSS Instantaneous Processing Software) developed at the University of Warmia and Mazury in Olsztyn (Paziewski, 2012). Below, observational equations of precise, relative positioning using singlefrequency double differenced (DD) carrier phase and pseudorange signals are provided. The model is used in GINPOS and it is presented for a single epoch and a particular double-difference of data from satellites $i$ and $j$ observed at $k$ and $l$ stations.

$$
\begin{gathered}
\lambda_{1} \phi_{k l, 1}^{i j}-\rho_{k l}^{i j}-\left(\alpha_{k}^{i} Z T D_{k}-\alpha_{k}^{j} Z T D_{k}-\alpha_{l}^{i} Z T D_{l}+\alpha_{l}^{j} Z T D_{l}\right)- \\
-\lambda_{1} N_{k l, 1}^{i j}=0 \\
P_{k l, 1}^{i j}-\rho_{k l}^{i j}-\left(\alpha_{k}^{i} Z T D_{k}-\alpha_{k}^{j} Z T D_{k}-\alpha_{l}^{i} Z T D_{l}+\alpha_{l}^{j} Z T D_{l}\right)=0
\end{gathered}
$$

Where:

$\lambda_{1} \varphi_{k l, 1}^{i j}$ - DD carrier phase (in meters);

$\rho_{k l}^{i j} \quad$ - DD geometric distance; note that DD geometric distance is a function of the station coordinates $\rho_{k l}^{i j}\left(x_{k}, y_{k}, z_{k}, x_{l}, y_{l}, z_{l}\right)$;

$N_{k l, 1}^{i j}$ - DD carrier phase ambiguity;

$\lambda_{1}$ - wavelength of L1/E1 frequency;

$\alpha_{k}^{i}$ - troposphere mapping function coefficient;

$Z T D_{k}$ - tropospheric zenith total delay;

$P_{k l, 1}^{i j}$ - double-differenced pseudorange.

The observation equations after linearization contain three groups of unknown parameters. These parameters are corrections to a priori station coordinates (both reference stations and rovers), DD (double differenced) ambiguities and corrections to a priori ZTDs (zenith tropospheric delay) for each site. The observational model is filtered with the least squares adjustment with constraints (Xu, 2007). Therefore, the total observational model is composed of two groups of observation equations: original observational equations (eq. 1) and the pseudoobservation equations (constraint equations). This allow for introducing configurable weights for a priori parameters. In the developed algorithms, the station coordinates and zenith tropospheric delays are stochastically constrained. In the examples presented in this paper, ZTD a priori values were tightly constrained since in ultra-rapid positioning and small networks it is extremely difficult to reliable estimate ZTD corrections (Wielgosz et al., 2011a, 2011b, 2011c; Paziewski, 2012). In such a case a priori ZTD may be obtained from any external source, e.g., from models presented by Leandro et al. (2008) or Bosy et al. (2010).

The presented observational model is applied for tightly combined (mixed) Galileo+EGNOS observations. In this concept, a single reference satellite is chosen for both of the systems in a particular session, namely DD observations are created using signals from different satellite systems. When combining signals from several satellite systems, one must take into consideration different time scales, coordinate systems and inter system biases (Wanninger and Wallstab-Freitag, 2007; Odijk et al., 2012). Although Galileo and EGNOS systems work in different coordinate reference frames (Galileo Terrestrial Reference Frame and EGNOS Terrestrial Reference Frame, respectively), both of the frames are the realizations of ITRS (International Terrestrial Reference System). Differences between the frames are on $\mathrm{cm}$ level and can be regarded as negligible for short baselines (EGN-SSD SoL V1.0, 2011). The difference in the time scales are canceled when double differenced observations are created. On the other hand, inter system bias (ISB), which is the receiver hardware delay, is introduced when mixed observations are processed (Hegarty et al., 2004). Nevertheless, research show that this bias is common for the same type of receivers and is relatively stable in time (Odijk et al., 2012). Thus, when observations are collected with the same type of receiver, this bias is cancelled out during differencing of observations. In other case it should be modeled.

\section{EXPERIMENT DESIGN}

\subsection{PROCESSING SCENARIO}

Observational data were collected with Septentrio TUR-N receiver using signals from SPIRENT GSS7700/7800 hardware multi-GNSS simulator at ESTEC/ESA. Simulations were performed for a reference station - KONI and for a simulated rover $-\mathrm{KK} 16$, with the distance between both stations of $\sim 31 \mathrm{~km}$. The length of the simulated observational session was $\sim 3.5 \mathrm{~h}(11: 30-$ 14:55 UTC). The location of the KONI station reflects position of real ASG-EUPOS station and the simulated baseline presents distance that is representative for this reference network. ASGEUPOS is Polish national active reference station system (Bosy et al., 2007).

Since the atmospheric models applied in the GNSS hardware simulator are rather simple and provide overly optimistic conditions for signal 

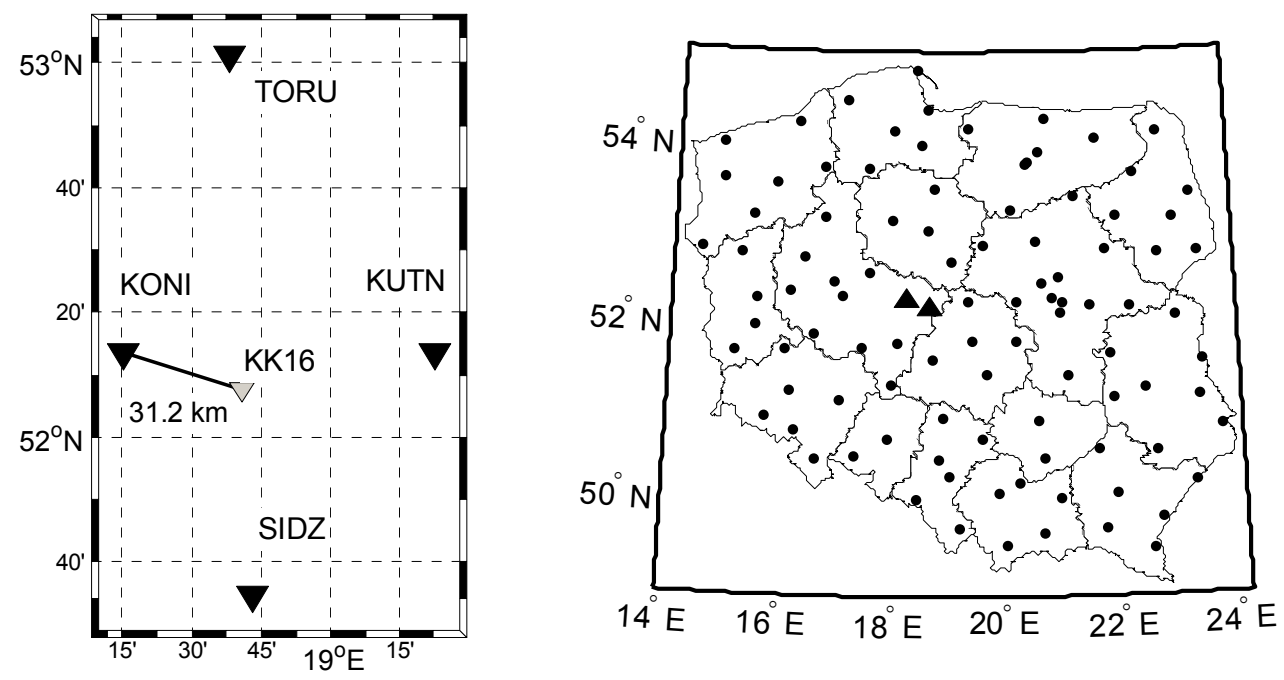

Fig. 1 Location of the processed baseline inside the ASG-EUPOS network.

propagation, simulated observational tropospheric and ionospheric delays were derived from more reliable external sources. In order to introduce the tropospheric delays into simulated „,clean” data, real zenith tropospheric delays obtained from the official reference network solution for Polish territory were used. A troposphere global mapping function - GMF was applied for the mapping of the zenith delays into slant ones (Boehm et al., 2006). Also, actual Global Ionosphere Maps (GIM) provided by the NASA JPL (Jet Propulsion Laboratory) from the GAIM (Global Assimilative Ionospheric Model) served as the basis for the computation of realistic ionospheric delays (Mandrake et al., 2005). The GIMs have spatial resolution of $1^{\circ} \times 1^{\circ}$ (longitude and latitude) and temporal resolution of 15 minutes.

The processing scenario assumed using singlefrequency $(\mathrm{E} 1 / \mathrm{L} 1$ for Galileo and EGNOS, respectively) carrier phase and pseudorange observations in double-differenced (relative) mode. The whole observing session was divided into 410 single-epoch sessions used in the processing. Please note that each of these single-epoch sessions was resolved independently, without accumulated information from previous epochs. This approach can be termed as an instantaneous positioning ( $\mathrm{Hu}$ et al., 2005). Reliable solution based on single-epoch data is extremely difficult and challenging task due to low number of observations and the lack of satellite geometry change (Kashani et al., 2007; Cellmer et al., 2010; Paziewski, 2012). Instantaneous positioning was selected in the presented analyses because of its challenging properties which better reflects differences between the tested positioning approaches.

Calculations were performed in the single baseline mode (baseline length $\sim 31 \mathrm{~km}$ ), KK16 served as rover station (Fig. 1). In such scenario, it is recommended to apply ionospheric corrections derived from the reference network solution (Wielgosz, et al. 2008), ionosphere maps (Schaer, 1999) or simple empirical ionosphere models like Klobuchar or NeQuick (Klobuchar, 1987; Di Giovanni and Radicella, 1990). However, as recent research show, these methods may be insufficient for high ionospheric activity, thus monitoring of the ionospheric disturbances may be required (Sieradzki et al., 2013). The analyzed scenario assumed processing with the use of a ionosphere model. Namely, the original ionospheric delays introduced from the GAIM model were reduced by $33 \%$ to reflect the application of the ionosphere model. It is known that simple ionosphere models like Klobuchar reduce the ionospheric delays to 50-60\% (Teunissen and Kleusberg, 1998). The simulated reduction by only $33 \%$ intentionally introduced more severe and challenging conditions for the processing algorithms. The influence of the tropospheric delay was reduced by the application of UNB3m model (Leandro, et al. 2008). It should be noted that this processing scenario can be regarded as difficult (instantaneous solution using single-frequency observations to process a single baseline of $31 \mathrm{~km}$ without network corrections). In practice, for that length of baselines, dual-frequency observations are used instead of single-frequency ones. However, this harsh processing scenario helps to underline differences between the analyzed and verified processing scenarios.

Broadcast orbits were used to calculate coordinates and clock corrections of the satellites. LAMBDA method was applied for the ambiguity resolution (Teunissen, 1995). W-ratio test was adopted for ambiguity resolution validation (Wang et al., 1998).

Special attention was paid to the performance of the carrier phase ambiguity resolution. The ambiguity resolution and validation success rate (AVSR) 


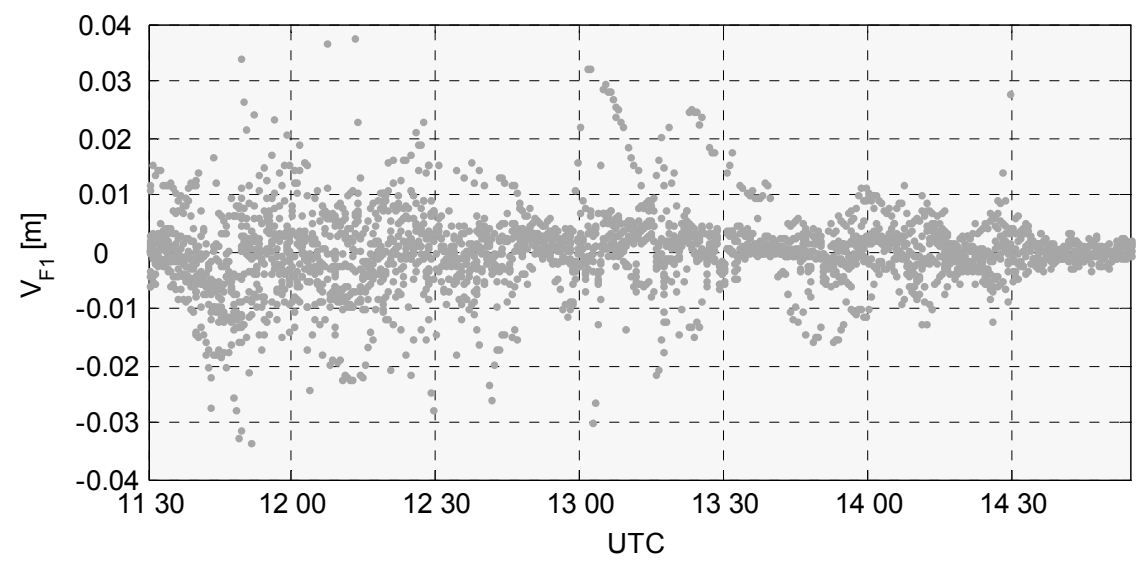

Fig. 2 Residuals of the DD carrier phase observations obtained with Galileo solution.

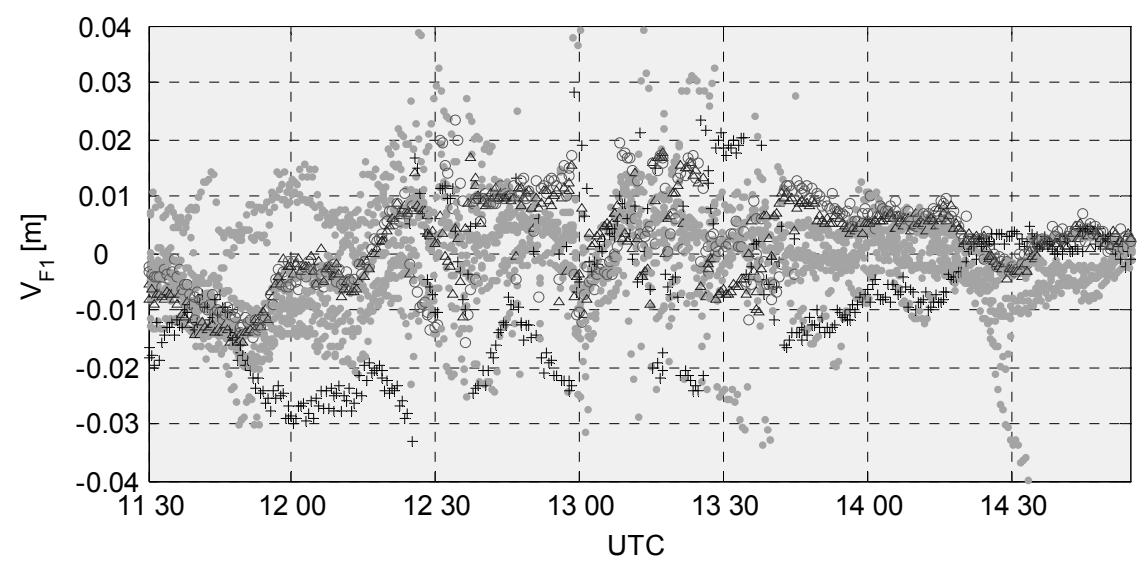

Fig. 3 Residuals of the DD carrier phase observations obtained with combined Galileo+EGNOS solution.

together with the ambiguity resolution and validation failure rate (AVFR) served as the indicators of the reliability of the solution. AVSR was defined as the ratio of the number of epochs with correctly resolved and validated ambiguities to the number of all epochs. AVFR was defined as the ratio of epochs with incorrectly resolved ambiguities which, however passed the validation test, to the number of all epochs. Additionally, standard deviations of the derived rover's coordinates (repeatability) were computed. This statistics were computed on the basis of correctly resolved and validated epochs.

\subsection{ANALYSIS ON THE ADJUSTED OBSERVATION RESIDUALS}

The applicability of the EGNOS range observations was analyzed on the basis of results of several processing scenarios. Firstly, in order to check if Galileo and EGNOS observations represent similar accuracy, their residuals were analyzed. This test allows for adoption of a priori standard deviations of Galileo and EGNOS observations in positioning experiment. Figures 2 and 3 present residuals of the
DD L1/E1 phase observations obtained with Galileo only (Fig. 2) and combined Galileo and EGNOS (Fig. 3) solution. These residuals relate to solution with resolved ambiguities. It should be noted that a priori standard deviations (sigma) of carrier phase and pseudorange observations for the Galileo and EGNOS signals were equal. On this basis, taking into account the mathematical correlations between observations and the satellite elevation angles, the respective stochastic model for DD observations was derived (Paziewski, 2012). Residuals of Galileo DD phase observations were marked with grey dots (Figs. 2, 3). Residuals of DD observations between Galileo and: EGNOS PRN 120, PRN 124, PRN 126 observations were marked with black crosses, black triangles and grey circles, respectively (Fig. 3). For Galileo solution, the majority of the residuals were smaller than $1 \mathrm{~cm}$, with some number of outliers (Fig 2). The analysis of the residuals of the mixed Galileo+EGNOS DD phase observations indicates that these observations were characterized by higher values (Fig. 3). 

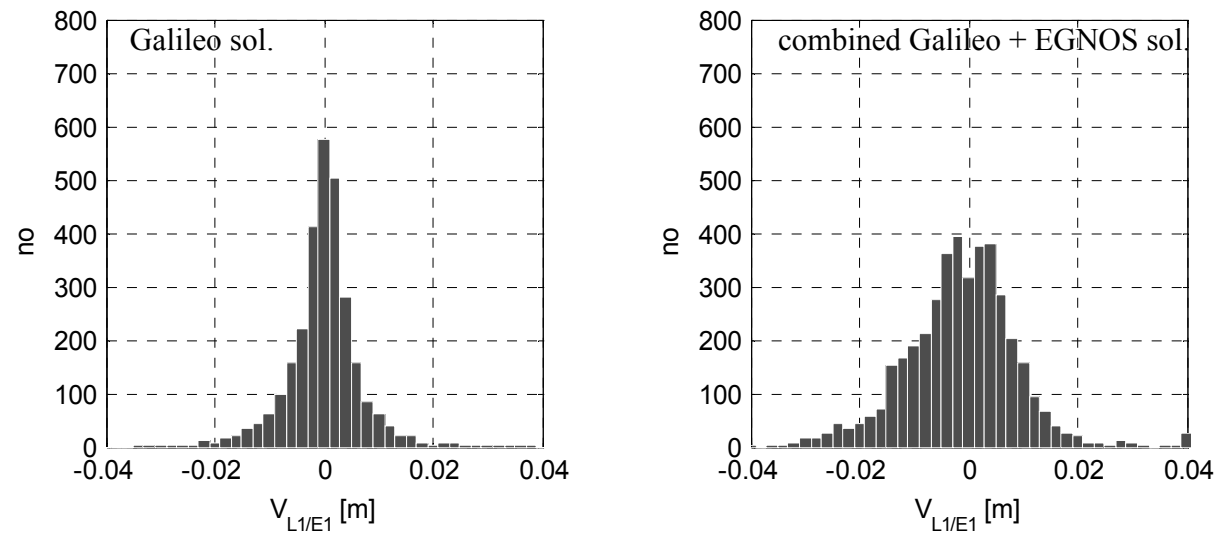

Fig. 4 Histograms of the DD phase residuals obtained with Galileo only solution (left), and with mixed Galileo and EGNOS solution (right).
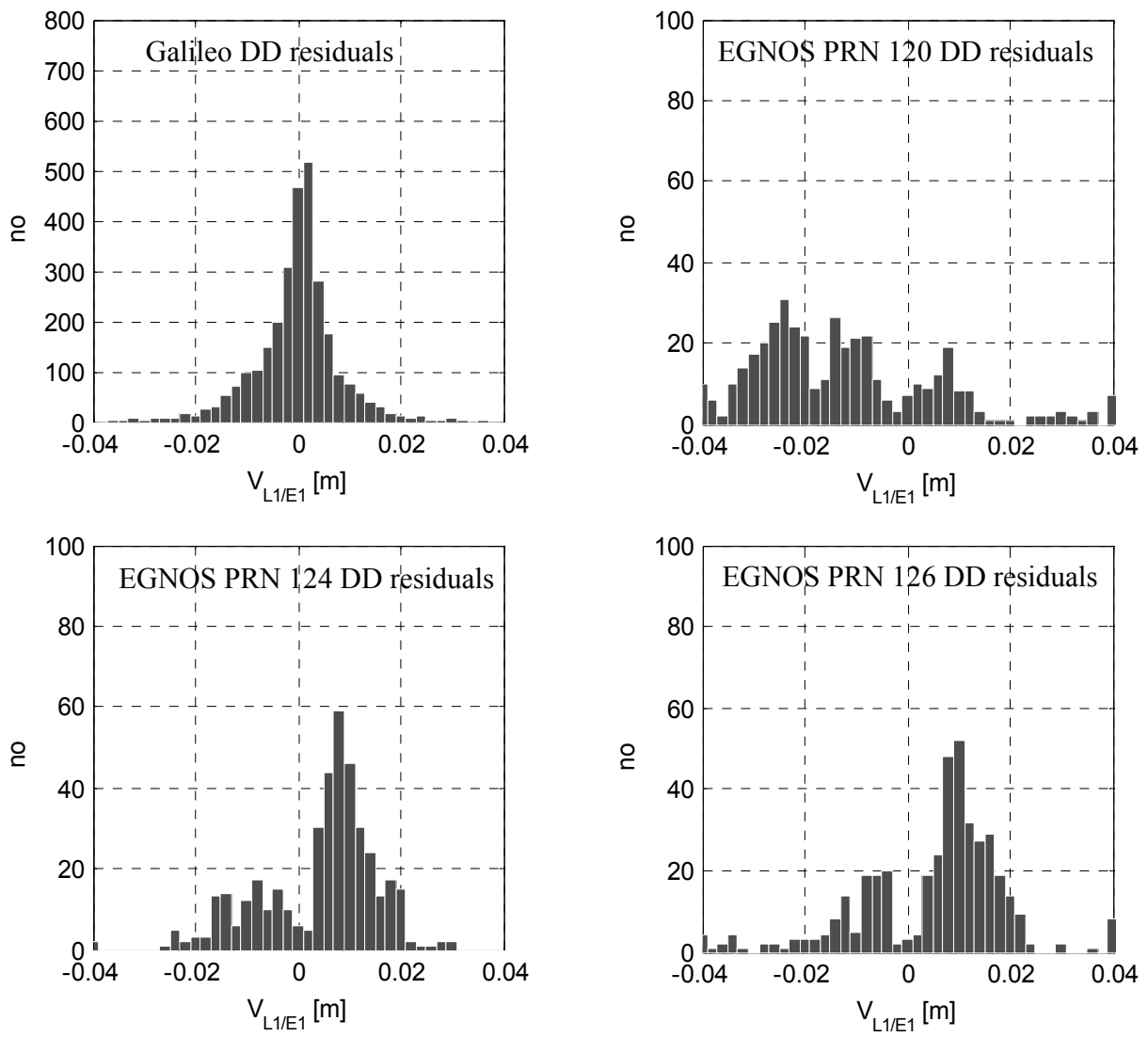

Fig. 5 Histograms of the DD phase residuals obtained with mixed Galileo and EGNOS solution grouped into: Galileo, EGNOS PRN 120, EGNOS PRN 124, EGNOS PRN 126 observations (with Galileo reference satellite).

The DD phase residual histograms for the Galileo and combined Galileo+EGNOS solutions can be found in Figure 4. It can be easily seen that combining observations from two systems (right panel) lead to higher residuals with respect to Galileo solution (left panel).

Figure 5 presents DD phase residuals grouped into four histograms: Galileo, EGNOS PRN 120,
EGNOS PRN 124, EGNOS PRN 126. During the whole session, a single Galileo satellite served as the reference (pivot) satellite. Residuals of the DD Galileo observations show symmetrical distribution. On the contrary, residuals of the EGNOS observations are characterized by systematic shift (bias). For the DD observations between Galileo and EGNOS PRN 120 the bias amounted to approximately $-2 \mathrm{~cm}$, for 
Table 1 Analyzed processing scenarios.

\begin{tabular}{|c|c|c|c|}
\hline Scenario \# & $\begin{array}{c}\text { number of Galileo } \\
\text { satellites }\end{array}$ & $\begin{array}{c}\text { numer of EGNOS } \\
\text { satellites }\end{array}$ & a priori sigma for EGNOS observations \\
\hline 1 & All observed & 0 & - \\
\hline $2 \mathrm{a}$ & All observed & 3 & $m_{o b s E G N}=m_{o b s G A L}$ \\
\hline $2 \mathrm{~b}$ & All observed & 3 & $m_{o b s E G N}=5 \times m_{o b s G A L}$ \\
\hline 3 & 5 & 0 & - \\
\hline 4 & 5 & 3 & $m_{o b s E G N}=5 \times m_{o b s G A L}$ \\
\hline
\end{tabular}

Table 2 Coordinate standard deviations (STD), mean of coordinate residuals $(\mathrm{dN}, \mathrm{dE}, \mathrm{dH})$, ambiguity resolution and validation success rate (AVSR) and ambiguity validation failure rate (AVFR) obtained with the analyzed scenarios.

\begin{tabular}{|c|c|c|c|c|c|c|c|c|c|}
\hline \multirow{2}{*}{ \# } & \multirow{2}{*}{ Scenario } & \multicolumn{2}{|c|}{$\mathrm{N}[\mathrm{m}]$} & \multicolumn{2}{|c|}{$\mathrm{E}[\mathrm{m}]$} & \multicolumn{2}{|c|}{$\mathrm{H}[\mathrm{m}]$} & \multirow{2}{*}{$\begin{array}{c}\text { AVSR } \\
{[\%]}\end{array}$} & \multirow{2}{*}{$\begin{array}{c}\text { AVFR } \\
{[\%]}\end{array}$} \\
\hline & & $\mathrm{dN}$ & STD & $\mathrm{dE}$ & STD & $\mathrm{dH}$ & STD & & \\
\hline 1 & GAL & 0.013 & 0.003 & -0.027 & 0.005 & 0.005 & 0.010 & 44.9 & 38.5 \\
\hline $2 \mathrm{a}$ & $\mathrm{GAL}+\mathrm{EGN}$ & 0.011 & 0.005 & -0.029 & 0.004 & 0.006 & 0.008 & 17.8 & 0.2 \\
\hline $2 b$ & $\begin{array}{c}\mathrm{GAL}+\mathrm{EGN} \\
\mathrm{m}_{\mathrm{obs} \mathrm{EGN}}=5 \mathrm{~m}_{\mathrm{obs} \mathrm{GAL}}\end{array}$ & 0.012 & 0.003 & -0.027 & 0.005 & 0.005 & 0.009 & 64.1 & 2.9 \\
\hline 3 & 5 sat. GAL & 0.012 & 0.009 & -0.034 & 0.012 & 0.034 & 0.147 & 17.6 & 47.6 \\
\hline 4 & $\begin{array}{l}5 \text { sat. GAL+EGN } \\
\mathrm{m}_{\text {obs EGN }}=5 \mathrm{~m}_{\text {obs GAL }}\end{array}$ & 0.016 & 0.005 & -0.040 & 0.005 & 0.013 & 0.008 & 15.1 & 4.9 \\
\hline
\end{tabular}

EGNOS PRN 124 and 126 this value reached approximately $1 \mathrm{~cm}$ (Fig. 5). These systematic biases in the observation residuals may be caused by not fully eliminated tropospheric and ionospheric delays. Please note that EGNOS satellites are almost stationary in respect to the receiver on the Earth. Moreover, EGNOS satellites were observed at low elevations $\left(22^{\circ}, 30^{\circ}, 30^{\circ}\right.$ for PRN 120, 124, 126, respectively). These conditions may cause stable in time values of the DD propagation errors, since only the reference Galileo satellite changes its position. Low elevations of EGNOS satellites cause also higher values of the residual ionospheric and tropospheric delays.

\subsection{PROCESSING SCENARIOS}

In order to analyze the applicability of the EGNOS observations to precise positioning 5 different processing scenarios were tested. First three scenarios assume good observing conditions with full visibility of Galileo satellites (elevation mask $10^{\circ}$ ). The next two scenarios assumed limited visibility of the Galileo satellites - only five Galileo satellites were visible (chosen from the all observed with the highest PDOP value).

Scenarios 1) and 3) assumed using only Galileo satellite data. Scenarios 2a), 2b) and 4) assumed tightly combined Galileo and EGNOS processing, but with different a priori sigma set for EGNOS observations (Table 1). Since the DD observations based on EGNOS signals show grater residuals (Figs. 3, 4, 5), in the strategies $2 \mathrm{~b}$ and 4 a scaling factor for a priori sigma of EGNOS signals was introduced. Three different values of the scaling factor were tested $(2.5,5,10)$, however in the paper we presented results obtained with the sigma scaling factor at 5, which gave the best results.

\section{RESULTS}

In this section, the results of processing of the tested scenarios are presented. Detailed statistics of the results are presented in Table 2. Application of scenario 1 gave in the result almost half of the epochs with correctly resolved and validated epochs (AVSR $=44.9 \%$ ). This value is not high, but note that these statistics relate to the single epoch and singlefrequency solution of $31 \mathrm{~km}$ baseline. In this scenario, also high AVFR was obtained (38.5\%), which indicates of low reliability of the solution. Introduction of the EGNOS signals (scenario 2a) resulted in the advancement of the ambiguity validation. Although lower number of AVSR was obtained $(17.8 \%)$, at the same time only $0.2 \%$ of the epochs was classified as incorrectly resolved and wrongly validated (AVFR statistics, Table 2). The best results regarding the ambiguity resolution were achieved for scenario $2 \mathrm{~b}$, when a priori sigma for EGNOS signals was 5 times higher than for Galileo 


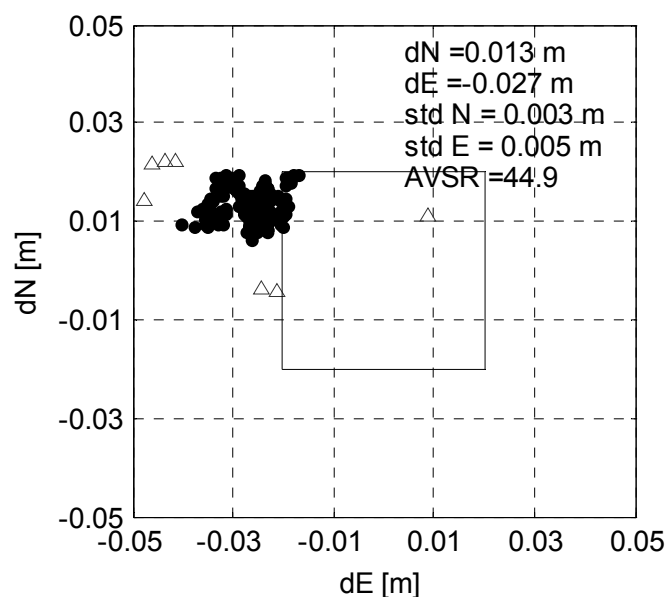

Fig. 6 Rover horizontal coordinate residuals obtained for scenario 1 .

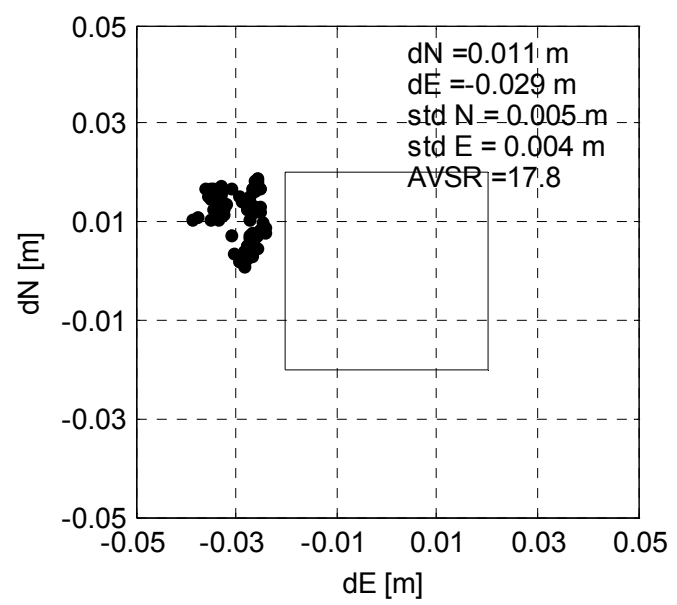

Fig. 8 Rover horizontal coordinate residuals obtained for scenario $2 \mathrm{a}$.

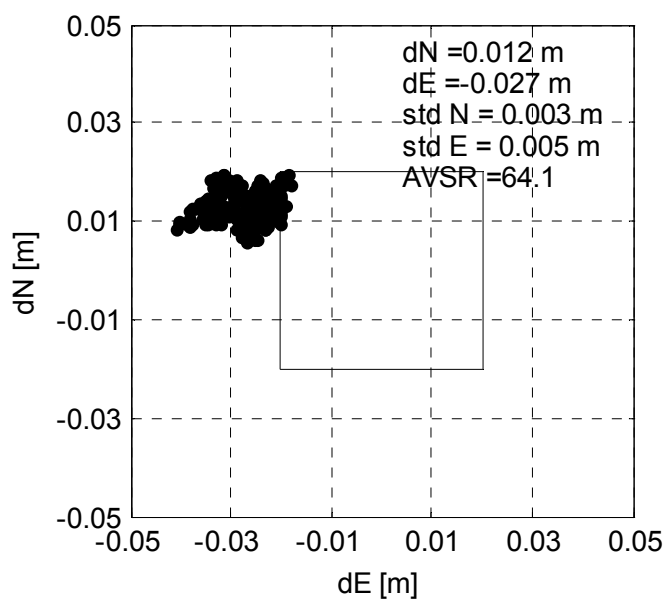

Fig. 10 Rover horizontal coordinate residuals obtained for scenario $2 b$.

ones. This allowed the filter to give higher residual to EGNOS observations. In this scenario $64.1 \%$ of the processed epochs were correctly resolved and validated and only $2.9 \%$ epochs were included to AVFR statistics. Mean residuals and standard deviations of the rover coordinates show similar

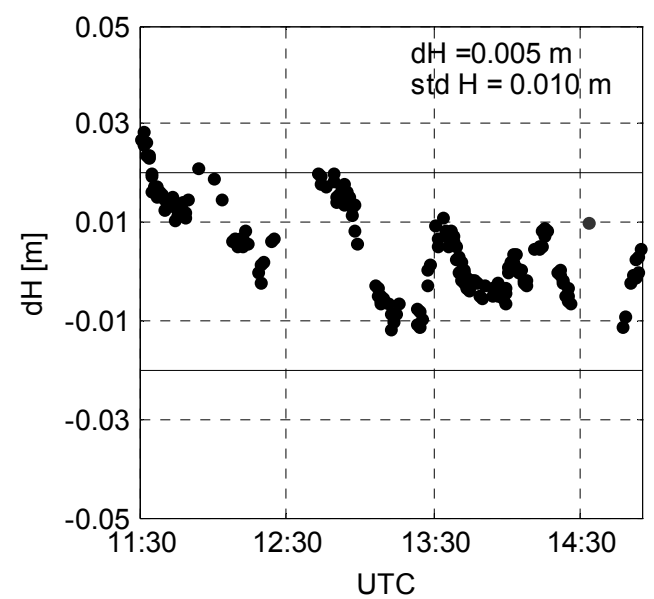

Fig. 7 Rover height residuals obtained for scenario 1 .

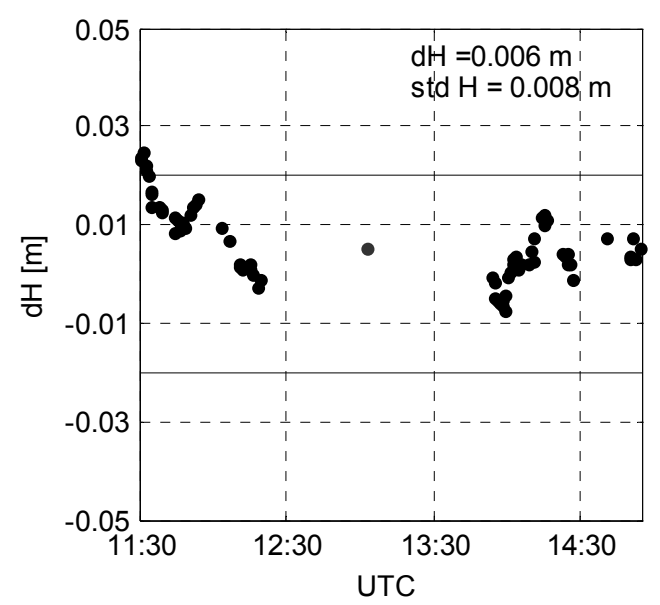

Fig. 9 Rover height residuals obtained for scenario $2 \mathrm{a}$.

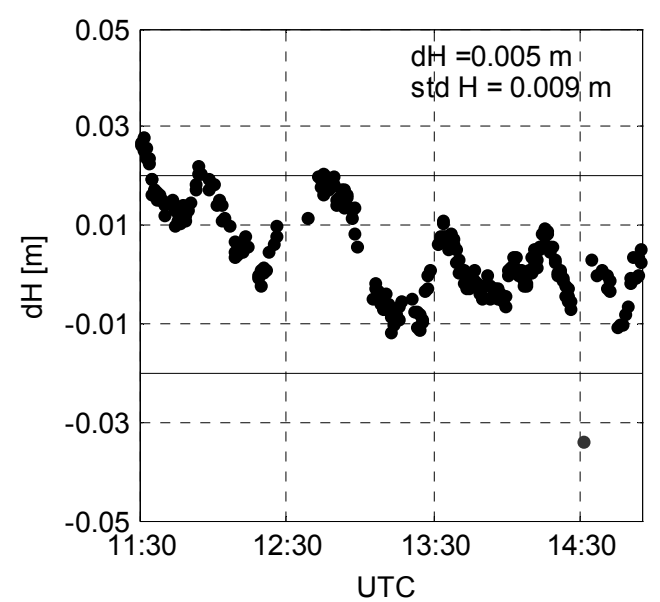

Fig. 11 Rover height residuals obtained for scenario $2 b$.

values in all scenarios (Table 2). Standard deviations of the horizontal coordinates did not exceed $0.6 \mathrm{~cm}$ for north and east components, respectively, and $1.0 \mathrm{~cm}$ for the height components.

For scenarios 3 and 4 with limited visibility of the Galileo satellites, the results were significantly 


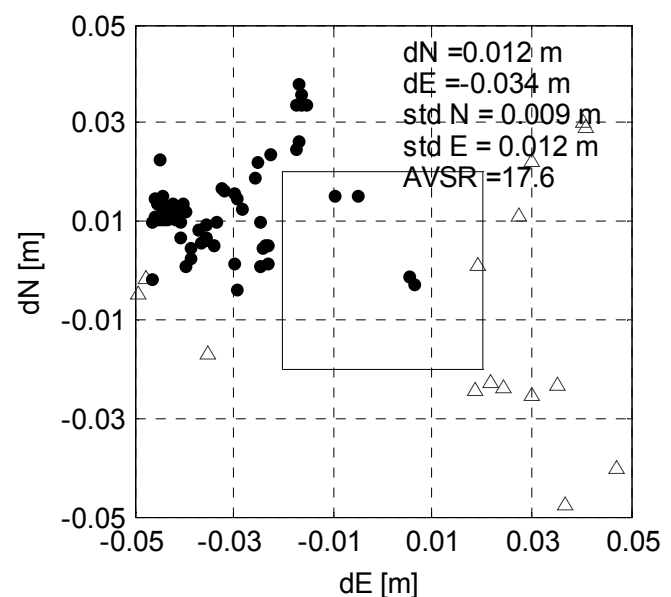

Fig. 12 Rover horizontal coordinate residuals obtained for scenario 3

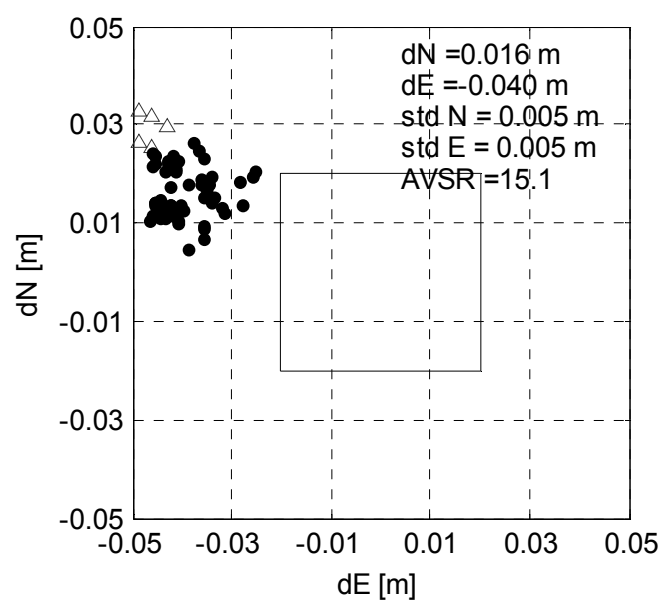

Fig. 14 Rover horizontal coordinate residuals obtained for scenario 4

worse. Please note that these conditions can be regarded as harsh. For scenario 3 assuming visibility of only 5 Galileo satellites, clearly greater number of epochs classified as AVFR than AVSR was obtained. Almost half of the epochs had incorrectly resolved ambiguities, which, however were not detected (AVFR $47.6 \%$ ). Introduction of signals from EGNOS satellites with lower a priori sigma (scenario 4) leads to improvement in AVFR statistics (4.9\%). Still, the rate of correctly resolved and validated epochs was low (AVSR $15.1 \%$ ). However the ambiguity resolution was more reliable (lower AVFR). Also the standard deviations of resulting coordinates decreased significantly, especially for the height component (from $0.147 \mathrm{~m}$ to $0.008 \mathrm{~m}$ ).

Figures 6-11 present rover coordinate residuals in local horizontal reference frame obtained with processing scenarios: 1, 2a, 2b. These processing scenarios relate to the unobstructed visibility of Galileo satellites. Coordinate residuals were computed with respect to the true coordinates of the rover (set up in the simulator scenario). The residuals obtained from solution classified as correctly resolved and validated (AVRS statistics) are marked with black

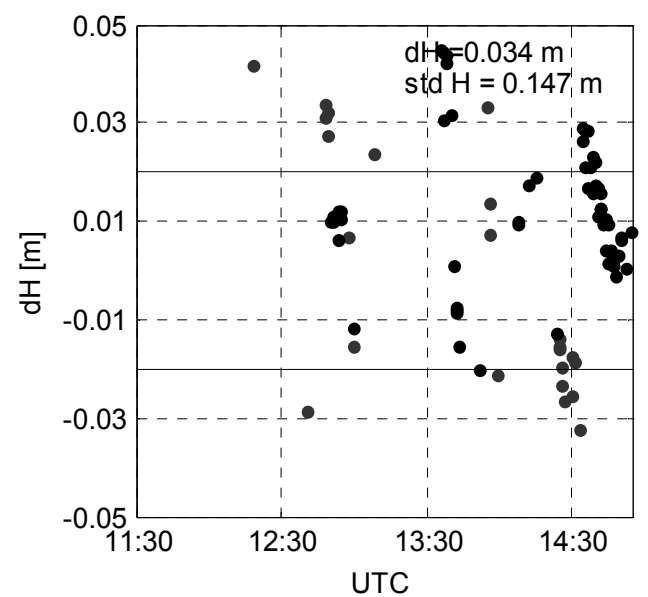

Fig. 13 Rover height residuals obtained for scenario 3

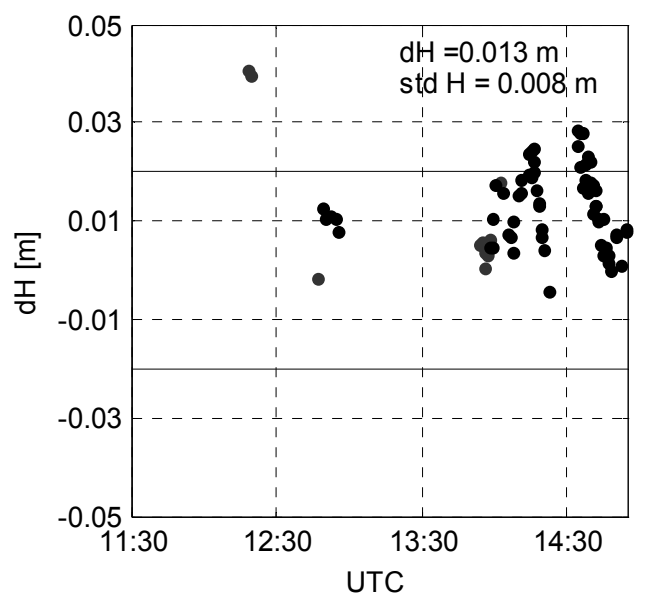

Fig. 15 Rover height residuals obtained for scenario 4

dots, alternatively residuals obtained with solutions classified to ambiguity validation failure (AVFR) are marked with triangles. A clear shift of the horizontal residuals with respect to the true position $(\mathrm{dE} \sim 3 \mathrm{~cm})$ may be caused by the effect of ionosphere-induced baseline shortening (Schaer, 1999), since ionospheric delay was not fully eliminated.

As it can be seen in the figures, the solutions for scenarios 1, 2a, 2b shows similar repeatability. However, a lower number of the resolved epochs for scenario $2 \mathrm{a}$ is clearly seen.

The rover coordinate residuals obtained for scenarios 3 and 4 for limited visibility of the satellites are shown in Figures $12-15$. It is clearly visible that solution obtained in scenario 3 is of very poor quality. There are many incorrectly resolved epochs (Fig. 12). Introduction of additional EGNOS signals (scenario 4), helped to detect epochs with incorrectly resolved ambiguities (Fig. 14).

\section{SUMMARY}

Judging on the basis of the simulated data, the application of additional observations from SBAS system may have positive impact on the performance 
of precise positioning, especially in case of limited satellite visibility. The greatest improvement was observed in the reliability of the solution (correctness of the validation procedure).

The main drawback of EGNOS data is that EGNOS satellites are stationary and observed at low elevations by the user in Central Europe, which cause higher values of the propagation errors. Solution on single frequency observations not supported with external corrections did not allow for highly successfully elimination of the atmospheric delays. This resulted in the higher residuals of the DD observations formed with EGNOS signals and forced to carefully set the weights (a priori sigmas) for SBAS and GNSS observations.

\section{ACKNOWLEDGMENTS}

This research was supported by grant agreed by the decision DEC-2011/03/N/ST10/05317 from Polish National Center of Science. The authors would like to thank Jaron Samson from ESTEC/ESA for opportunity to use GNSS signals simulator.

\section{REFERENCES}

Boehm, J., Niell, A., Tregoning, P. and Schuh, H.: 2006, Global Mapping Function (GMF): A new empirical mapping function based on numerical weather model data, Geophys. Res. Lett., 33, L07304. DOI: $10.1029 / 2005$ GL025546

Bosy, J., Graszka, W. and Leonczyk, M.: 2007.: ASGEUPOS - a multifunctional precise satellite positroning system in Poland, Eur. J. Nav., 5(4), 2-6.

Bosy, J., Rohm, W. and Sierny, J.: 2010.: The concept of the near real time atmosphere model based on the GNSS and the meteorological data from the ASG-EUPOS reference stations, Acta Geodyn. Geomater., 7, No. 3(159), 253-261.

Cacoń, S., Kapłon, J., Kontny, B., Weigel, J., Švábenský, O. and Kopecký J.: 2010, Recent local geodynamics in the central part of the Stołowe Mts., Acta Geodyn. Geomater., 7, No. 3 (159), 335-342.

Cellmer, S., Wielgosz, P. and Rzepecka, Z.: 2010, Modified Ambiguity Function Approach for GPS carrier phase positioning, J. Geod., 84, No 4, 267-275.

DOI: $10.1007 / \mathrm{s} 00190-009-0364-8$

Di Giovanni, G. and Radicella, S.M.: 1990, An analytical model of the electron density profile in the ionosphere, Advances in Space Research, 10(11), 27-30. DOI: $10.1016 / 0273-1177(90) 90301-F$

EGNOS Safety of Life Service Definition Document, 2011, Ref: EGN-SSD SoL, V1.0.

Giorgi, G., Teunissen, P.J.G. and Gourlay T.P.: 2012, Instantaneous global navigation satellite system (GNSS) based attitude determination for maritime applications, IEEE J. Ocean. Eng., 37, No. 3, 348362.

Hegarty, C., Powers, E. and Fonville, B.: 2004, Accounting for timing biases between GPS, modernized GPS, and Galileo signals, Proc. 36th Annual Precise Time and Time Interval (PTTI) Meeting, Washington, DC, 7-9 December, 307-317.

Hu, G., Abbey, D.A., Castleden, W.E., Earls, C., Ovstedal, O. and Weihing, D.: 2005, An approach for instantaneous ambiguity resolution for medium- to long-range multiple reference station networks, GPS Solut., 9, 1-11. DOI: 10.1007/s10291-004-0120-8

Leandro, R.F., Langley, R.B. and Santos, M.C.: 2008, UNB3m pack: A neutral atmosphere delay package for GNSS, GPS Solut., 12, No. 1, 65-70. DOI: 10.1007/s10291-007-0077-5

Kapłon, J. and Cacoń, S.: 2009, Research on the Marginal Sudetic fault activity with use of GPS and precise leveling techniques, Acta Geodyn. Geomater., 6, No. 3 (155), 323-329.

Kashani, I., Wielgosz, P., Grejner-Brzezinska, D.A. and Mader, G.L.: 2008, A New Network-Based RapidStatic Module for the NGS Online Positioning User Service - OPUS-RS, Navigation, 55, No. 3, 255-264. DOI: $10.1179 / 175227007 X 197156$

Klobuchar, J.: 1987, Ionospheric time-delay algorithms for single-frequency GPS users, IEEE Transactions on Aerospace and Electronic Systéme, (3), 325-331.

Mandrake, L., Wilson, B., Wang, C., Hajj, G., Mannucci, A. and Pi, X.: 2005, A performance evaluation of the operational Jet Propulsion Laboratory/University of Southern California Global Assimilation Ionospheric Model (JPL/USC GAIM), J. Geophys. Res., 110, A12306. DOI: 10.1029/2005JA011170

Odijk, D., Teunissen, P.J.G. and Huisman, L.: 2012, First Results of Mixed GPS + GIOVE Single-Frequency RTK in Australia, J. Spat. Sci., 57:1, 3-18. DOI: $10.1080 / 14498596.2012 .679247$

Paziewski, J.: 2012, New algorithms for precise positioning with use of Galileo and EGNOS European satellite navigation systems, $\mathrm{PhD}$ Dissertation, University of Warmia and Mazury in Olsztyn, (in Polish).

Sauer, K. and Ochieng, W.Y.: 2002, Integrated use of GPS and EGNOS carrier phase observations for high precision kinematic positioning - first experiences, J. Geospat. Eng., 4(1), 59-67.

Schaer, S.: 1999, Mapping and predicting Earth's ionosphere using global positioning system, $\mathrm{PhD}$ Dissertation, Astronomical Institute, University of Berne, Switzerland.

Schenk, V., Schenková, Z., Bosy, J. and Kontny, B.: 2010, Reliability of GPS data for geodynamic studies case study: Sudeten area, The Bohemian Massif, Acta Geodyn. Geomater., 7 No. 1 (157), 113-128.

Sieradzki, R., Czerniak, I. and Krankowski, A.: 2013, Nearreal time monitoring of the TEC fluctuations over the northern hemisphere using GNSS permanent networks, Advances in Space Research, 52(3), 391402. DOI: $10.1016 /$ j.asr.2013.03.036

Teunissen, P.J.G.: 1995, The least-squares ambiguity decorrelation adjustment: a method for fast GPS integer ambiguity estimation, J. Geod., 70, 65-82. DOI: $10.1007 / \mathrm{BF} 00863419$

Teunissen, P.J.G. and Kleusberg, A.: 1998, GPS for Geodesy $2^{\text {nd }}$ ed., Springer-Varlag, Berlin-Heidelberg.

Tiberius, C., Pany, T., Eissfeller, B., Joosten, P. and Verhagen, S.: 2002, 0.99999999 confidence ambiguity resolution with GPS and Galileo, GPS Solut., 6, 9699. DOI: $10.1007 / \mathrm{s} 10291-002-0022-6$

Wang, J., Stewart, M. and Tsakiri, M.: 1998, A discrimination test procedure for ambiguity resolution onthe-fly, J. Geod., 72, 644-653. DOI: $10.1007 / \mathrm{s} 001900050204$

Wanninger, L. and Wallstab-Freitag, S.: 2007, Combined Processing of GPS, GLONASS, and SBAS Code 
Phase and Carrier Phase Measurements, Proc. ION GNSS 2007, Fort Worth, Tx., Sep., 25-28, 866-875.

Wielgosz, P., Krankowski, A., Sieradzki, R., and GrejnerBrzezinska, D.A., 2008, Application of predictive regional ionosphere model to medium range RTK Positioning, Acta Geophys., 56, No 4, 1147-1161. DOI: $10.2478 / \mathrm{s} 11600-008-0059-1$

Wielgosz, P., Cellmer, S., Rzepecka, Z., Paziewski, J. and Grejner-Brzezinska, D.: 2011a, Troposphere modeling for precise GPS rapid static positioning in mountainous areas, Meas. Sci. Technol., 22, No. 4. DOI: $10.1088 / 0957-0233 / 22 / 4 / 045101$

Wielgosz, P., Paziewski, J. and Baryła, R.: 2011b, On constraining zenith tropospheric delays in processing of local GPS networks with Bernese software, Surv. Rev., 43, No 323.

DOI: $10.1179 / 003962611 X 13117748891877$

Wielgosz, P., Paziewski, J., Krankowski, A., Kroszczynski, K. and Figurski, M.: 2011c, Results of the application of tropospheric corrections from different troposphere models for precise GPS rapid static positioning, Acta Geophys., 60, No. 4, 1236-1257. DOI: $10.2478 / \mathrm{s} 11600-011-0078-1$

ESA 2006, EGNOS The European Geostationary Navigation Overlay System - A cornerstone of Galileo, In: Ventura-Traveset, J., Flament, D (eds) SP-1303, The Netherlands.

Verhagen, S.: 2002, Performance analysis of GPS, Galileo and integrated GPS-Galileo, Proc. of ION GPS 2002, 24-27 September 2002, Portland.

Xu, G.: 2007, GPS: Theory, Algorithms and Applications, 2nd ed. (Berlin Heidelberg Springer-Verlag).

Zhao, C., Ou, J. and Yuan, Y.: 2005, Positioning accuracy and reliability of GALILEO, integrated GPSGALILEO system based on single positioning model, Chi. Sci. Bull., 50, No. 12, 1252-1260. 